\title{
On a High Dimensional Riemann's Removability Theorem
}

\author{
Yukinobu Adachi ${ }^{1}$ \\ 1 2-12-29 Kurakuen, Nishinomiya, Hyogo, Japan \\ Correspondence: Yukinobu Adachi, 2-12-29 Kurakuen, Nishinomiya, Hyogo 662-0082, Japan. E-mail: \\ fwjh5864@nifty.com
}

Received: March 8, 2014 Accepted: April 16, 2014 Online Published: June 26, 2014

doi:10.5539/jmr.v6n3p8 URL: http://dx.doi.org/10.5539/jmr.v6n3p8

\begin{abstract}
Let $M$ be a (connected) complex manifold and $E$ be a closed capacity zero set. Let $X$ be a (connected) complex compact Kobayashi hyperbolic space whose universal covering space is Stein and let $f$ be a holomorphic map of $M-E$ to $X$. Then $f$ can be extended holomorphically to a map of $M$ to $X$.
\end{abstract}

Keywords: removable singularities, capacity zero set, compact Kobayashi hyperbolic space

2000 Mathmatics Subject Classification: Primary, 32H02; Secondary, 32Q45

\section{Introduction}

Nishino in 1979 proved the extension problem in the case that $\operatorname{dim} M=1, E$ is a closed logarithmic capacity zero set and $X$ is a compact Riemann surface whose genus is greater than 1 using the Ahlfors theory of covering surfaces. Masakazu Suzuki in 1987 gave the same result using a method of differential geometry and a result in a case that $E$ is a closed pluripolar set set in $M$ which is a domain $D$ in $\mathbf{C}^{m}$ with $m \geqq 1$ and $X$ is a manifold whose universal covering is a polynomial convex bounded domain in $\mathbf{C}^{n}$ in the case that the image of the map of $D-E$ is relatively copact in $X$ in (Suzuki, 1988) in the sequel.

We shall prove the case when $M$ is a complex manifold with an arbitrary dimension, $E$ is a closed capacity zero set in $M$ (see Definition 3.2) and $X$ is a compact Kobayashi hyperbolic space with arbitrary dimension whose universal covering space is Stein (Theorem 4.6) using the Kobayashi hyperbolic geometry and Fujimoto's theorem (Proposition 3.4) according to a properties owing Suzuki's method. Our results contain generalizations of Nishino and Suzuki theorems (Theorem 3.7, Corollary 3.8, Theorem 4.6 and Corollary 4.7). About this problem, see section 2.2 in (Noguchi,1993). And we give some appications (Corollary 4.8 and Theorem 4.9).

\section{Preliminary (1)}

We show the lemmas by the same way in (Suzuki, 1987). But we claim it independently to an existence of a holomorphic map $f$ of $M-E$ to $X$.

Let $\Delta$ be the unit disk in $z$-plane and $e$ be a compact set of $\Delta$ of logarithmic capacity zero. Let $g=\frac{4|d w|^{2}}{\left(1-|w|^{2}\right)^{2}}$ be a Poincaré metric of $\Delta$ in $w$-plane. Since the universal covering surface of $\Delta-e$ is $\Delta$, we set a hyperbolic metric of $\Delta-e$ as $h(z)|d z|^{2}=\left.\left(\left.\pi^{-1}\right|_{U}\right)^{*} g\right|_{V}$ where $U \subset \Delta-e, V \subset \Delta$ and $\pi(V)=U$ where $\pi$ is an universal covering map of $\Delta$ to $\Delta-e$.

From the Selberg theorem in (Selberg, 1937), there is a harmonic function $u$ on $\Delta-e$ such that $u=0$ on $\partial \Delta$ and $u(z) \rightarrow \infty$ when $z \in \Delta-e, z_{0} \in e$ and $z \rightarrow z_{0}$.

Set $C_{\lambda}:=\{u=\lambda\}(0 \leqq \lambda<\infty), D_{0}:=\{\delta \leqq u\} \cap(\Delta-e)$ and $D_{\lambda}:=\{\delta \leqq u \leqq \lambda\}$ such that $\delta$ is sufficiently small positive number. Then we may assume that $D_{\lambda}$ is connected, $C_{\lambda}$ consists of finite number of closed curve and $C_{\delta}$ is a Jordan closed curve near $C_{0}$. We fix such a constant $\delta$. When $d v(z)$ is a conjugate differential of $d u(z)$, we can assume that $\int_{C_{\lambda}} d v(z)=2 \pi$ by Selberg theorem.

We set $\left|D_{\lambda}\right|=\int_{\delta \leqq u \leqq \lambda} h(z) d u(z) d v(z)$ and $\left|C_{\lambda}\right|=\int_{u=\lambda} \sqrt{h(z)}|d z|$. Then following lemmas are proved. 
Lemma 2.1 (Lemma 2, Suzuki,1987) There exists a subsequence $\left\{\lambda_{v}\right\}_{v=1,2, \ldots}$ such that

$$
\lim _{v \rightarrow \infty} \frac{\left|C_{\lambda_{\nu}}\right|}{\left|D_{\lambda_{\nu}}\right|}=0, \lambda_{1}<\lambda_{2}<\cdots \rightarrow \infty .
$$

Proof. By the Schwarz Lemma,

$$
\left|C_{\lambda}\right|^{2} \leqq \int_{u=\lambda} d v \int_{u=\lambda} h(z) d v=2 \pi \frac{d}{d \lambda}\left|D_{\lambda}\right| .
$$

If we set $E_{\varepsilon}:=\left\{\lambda \in[1, \infty) ; \varepsilon\left|D_{\lambda}\right| \leqq\left|C_{\lambda}\right|\right\}$ for every small fixed $\varepsilon>0$,

$$
m e s\left(E_{\varepsilon}\right)=\int_{E_{\varepsilon}} d \lambda \leqq \frac{2 \pi}{\varepsilon^{2}} \int_{1}^{\infty} \frac{d\left|D_{\lambda}\right|}{\left|D_{\lambda}\right|^{2}}=\frac{2 \pi}{\varepsilon^{2}}\left(\frac{1}{\left|D_{1}\right|}-\lim _{\lambda \rightarrow \infty} \frac{1}{\left|D_{\lambda}\right|}\right)<\infty .
$$

Therefore, there are $\lambda_{1}<\lambda_{2}<\cdots \rightarrow \infty$ and $\varepsilon_{1}>\varepsilon_{2}>\cdots \rightarrow 0$ such that $\frac{\left|C_{\lambda_{\nu}}\right|}{\left|D_{\lambda_{\nu}}\right|}<\varepsilon_{\nu}$.

Lemma 2.2 (cf. Lemma 1, Suzuki, 1987) $\left|D_{\lambda}\right| \leqq 2\left|\partial D_{\lambda}\right|$.

Proof. Let a subdivision of $D_{\lambda}=\Sigma_{\mu=1}^{N} d_{\mu}$ and $\partial D_{\lambda}=\Sigma_{\mu=1}^{N} \partial d_{\mu}=\Sigma_{\tau=1}^{T} \partial d_{\tau}$ where $d_{\mu}$ is a small simply connected domain, $\partial d_{\tau}$ is a part of some $\partial d_{\mu}$ by cancellation and $h(z)|d z|^{2}=F_{\mu}^{*} g$ on $d_{\mu}$ where $F_{\mu}=\left.\pi^{-1}\right|_{\mu}$.

Then

$$
\begin{aligned}
\left|D_{\lambda}\right| & =\Sigma_{\mu} \int_{d_{\lambda}} d d^{c} \log \left(\frac{4\left|F_{\mu}^{\prime}(z)\right|^{2}}{\left(1-\left|F_{\mu}(z)\right|^{2}\right)^{2}}\right) \\
& =2 \Sigma_{\mu} \int_{\partial d_{\mu}} d^{c} \log \left(\frac{\left|F_{\mu}^{\prime}(z)\right|}{1-\left|F_{\mu}(z)\right|^{2}}\right) \\
& =2 \Sigma_{\mu}\left\{\int_{\partial d_{\mu}} d\left(\arg F_{\mu}^{\prime}(z)\right)-\int_{\partial d_{\mu}} d^{c} \log \left(1-\left|F_{\mu}(z)\right|^{2}\right)\right\} \\
& =-2 \Sigma_{\mu} \int_{\partial d_{\mu}} d^{c} \log \left(1-\left|F_{\mu}(z)\right|^{2}\right) \\
& =-2 \Sigma_{\tau} \int_{\partial d_{\tau}} d^{c} \log \left(1-\left|F_{\mu}(z)\right|^{2}\right) \leqq 2\left|\partial D_{\lambda}\right|,
\end{aligned}
$$

since $\left|d^{c} \log \left(1-|w|^{2}\right)\right|=\left|2 \operatorname{Im}\left(\frac{w d \bar{w}}{1-|w|^{2}}\right)\right| \leqq 2 \frac{|d w|}{1-|w|^{2}}$.

Lemma 2.3 Let $C_{\lambda_{\nu}}$ and $D_{\lambda_{\nu}}$ be same in Lemma 2.1. Then $\left|C_{\lambda_{\nu}}\right| \rightarrow 0(v \rightarrow \infty)$.

Proof. From Lemma 2.2,

$$
\frac{1}{2} \leqq \frac{\left|\partial D_{\lambda_{\nu}}\right|}{\left|D_{\lambda_{\nu}}\right|}=\frac{\left|C_{\delta}\right|+\left|C_{\lambda_{\nu}}\right|}{\left|D_{\lambda_{\nu}}\right|}
$$

Since $\lim _{\nu \rightarrow \infty} \frac{\left|C_{\lambda_{\nu}}\right|}{\left|D_{\lambda_{\nu}}\right|} \rightarrow 0$ and $\left|C_{\delta}\right|$ is a constant, $\left|D_{\lambda_{v}}\right|$ is uniformly bounded for $v$. Then we conclude that $\left|C_{\lambda_{\nu}}\right| \rightarrow$ $0(v \rightarrow \infty)$.

\section{Preliminary (2)}

In this section we denote that $M$ is a connected normal complex space with an arbitrary dimension (for its definition, see for example Nishino,2001, pp. 212, 267).

Definition 3.1 We denote $u \in P S H(M)$ when following conditions are satisfied;

(1) $u$ is an upper semicontinuous function on $M,-\infty \leqq u<\infty$ and $u \neq \equiv-\infty$.

(2) For any open set $W$ in $\mathbf{C}$ and any holomorphic map $\psi: W \rightarrow M, u \circ \psi$ is a subharmonic function on $W$ in the usal sence or identically equal to $-\infty$.

Definition 3.2 (cf. Definition 2.5, Fujimoto,1971) We define a subset $E$ of $M$ as a pluripolar set if we can take every point $p$ of $E$ such that for some $u_{p} \in P S H\left(U_{p}\right)$ where $U_{p}$ is a coordinate neighborhood of $p$ in $M$ such that $E \cap U_{p} \subset\left\{p \in U_{p} ; u_{p}=-\infty\right\}$. And we call $E^{\prime}$ a capacity zero set if we can take an at most countable family $\left\{E_{v}\right\}$ of pluripolar subsets of $M$ such as $E^{\prime}=\bigcup_{v} E_{v}$.

Remark 3.3 (Remark in Fujimoto, 1971, p. 3) A closed set in complex plane $\mathbf{C}$ is of capacity zero in the sence of above definition if and only if it is of logarithmic capacity zero in the usual sence. It is well known that an analytic subset of $M$ is a closed pluripolar set. 
Proposition 3.4 (Proposition 3.1, Fujimoto, 1971) Let $E$ be a closed capacity zero set in a normal space $M$ and $f \in O(M-E)$ be locally bounded on every point of $E$. Then $f \in O(M)$.

Corollary 3.5 (Corollary 3.2, Fujimoto, 1971) If E be the same as the above proposition, then $D-E$ is connected for any domain $D$ of $M$.

Remark 3.6 Since the concept of the capacity zero set contains the one of the pluripolar set, above results are established when $E$ is a closed pluripolar set.

Theorem 3.7 Let $E$ be a closed capacity zero set in a normal space $M$ and $f$ be a holomorphic map of $(M-E)$ to a space $X$ which may be open or compact. If for every point of $p \in E$ there is a neighborhood of $U$ of $p$ and a Stein subdomain $X_{0}$ of $X$ such that $f(U-E) \Subset X_{0}$ where $X_{0}$ may be depend on $U-E$, then $f$ is extended holomorphically to a map of $M$ to $X$.

Proof. Since $X_{0}$ is Stein, there is an analytic polyhedron $\mathcal{P}$ of $X_{0}$ which contains $f(U-E)$. By Oka-Grauert theorem (see for example (Nishino, 2001, Theorem 8.1)), $\mathcal{P}$ is imbedded to a polydisk of $\mathbf{C}^{N}$ as a normal analytic subset, then above claim is true from Proposition 3.4.

Since the open Riemann surface is Stein, the following corollary is obtained.

Corollary 3.8 Let $M$ and $E$ be the same as in Theorem 3.7. If $f$ is a holomorphic map of $M-E$ to any open or compact Riemann surface $R$ with $f(M-E) \Subset R$ where genus $g$ of $R$ satisfies $0 \leqq g \leqq \infty, f$ can be extended holomorphically to a map of $M$ to $R$.

\section{Conclusion and Applications}

We use the word "hyperbolic" as "Kobayashi hyperbolic". For the concept of Kobayashi hyperbolicity and its properties, see for example (Kobayashi, 2005) which is a manifold version and (Kobayashi, 1998) which is a space version.

Proposition 4.1 Let $\Delta$ be the unit disk in z-plane and e be a compact set of logarithmic capacity zero. Let X be an open or compact hyperbolic space with an arbitrary dimension such that for every connected compact set $K \Subset X$ there is a holomorphically separable function on a neighborhood of $K$. Then if $f$ is a holomorphic map of $\Delta-e$ to $X$ such that $f(\Delta-e)$ is relatively compact in $X$, in particular if $X$ is compact, then $f$ can be extended holomorphically to a map of $\Delta$ to $X$.

Proof. Let $z_{0}$ be an arbitrary point of $e$ and $C_{\lambda_{v}}$ is the same of Lemma 2.3. We write $\lambda_{v}$ to $v$ anew. Let $C_{1}^{0}$ be one of $C_{1}$ which surrounds $z_{0}$ and $C_{2}^{1}$ consits of $C_{2}$ surrounded by $C_{1}^{0}$. Let $C_{2}^{0}$ be one of $C_{2}^{1}$ which surrounds $z_{0}$ (we may assume that $C_{v}^{0}$ is a Jordan closed curve). We define inductively $C_{v}^{0}$ and $C_{v+1}^{1}$ such the method and $E_{v}$ is a domain surrounded by $C_{v}^{0}$ as the outer boundary and $C_{v+1}^{1}$ as the inner boundary. From Corollary 3.5, there is a $z_{v} \in C_{v}^{0}$ with $z_{v} \rightarrow z_{0}(v \rightarrow \infty)$. When we take a subsequence that $\left\{z_{v}\right\}$ if necessary, we may assume that $f\left(z_{v}\right) \rightarrow r \in X(v \rightarrow \infty)$ because $f(\Delta-e)$ is relatively compact in $X$.

Let $z_{v}^{\prime}$ be an arbitrary point of $C_{v}^{0}$. Then $d_{X}\left(f\left(z_{v}^{\prime}\right), r\right) \leqq d_{X}\left(f\left(z_{v}\right), r\right)+d_{X}\left(f\left(z_{v}\right), f\left(z_{v}^{\prime}\right)\right) \leqq d_{X}\left(f\left(z_{v}\right), r\right)+d_{\Delta-e}\left(z_{v}, z_{v}^{\prime}\right) \leqq$ $d_{X}\left(f\left(z_{v}\right), r\right)+\left|C_{v}\right| \rightarrow 0(v \rightarrow \infty)$. Let $z_{v+1}^{\prime}$ be an arbitrary point of $C_{v+1}^{1}$. Then $d_{X}\left(f\left(z_{v+1}^{\prime}\right), r\right) \leqq d_{X}\left(f\left(z_{v+1}\right), r\right)+$ $d_{X}\left(f\left(z_{v+1}\right), f\left(z_{v+1}^{\prime}\right)\right) \leqq d_{X}\left(f\left(z_{v+1}\right), r\right)+d_{\Delta-e}\left(z_{v+1}, z_{v+1}^{\prime}\right) \leqq d_{X}\left(f\left(z_{v+1}\right), r\right)+\left|C_{v+1}\right| \rightarrow 0(v \rightarrow \infty)$.

Therefore if we give an arbitrarily small positive number $\varepsilon$, there is a large integer $N_{\varepsilon}$ such that $\left\|f\left(\partial E_{\gamma}\right)-r\right\|<\varepsilon$ for $v \geqq N_{\varepsilon}$ depending a number $\varepsilon$. We assume that there is a point $z_{v}^{\prime} \in E_{v}$ such that $\left\|f\left(z_{v}^{\prime}\right)-s\right\|<\varepsilon$ for $v \geqq M_{\varepsilon}$ which is a large integer depending a number $\varepsilon$ where $s$ is a point of $X$ with $r \neq s$. And $f\left(\bar{E}_{v}\right)$ is compact with $f\left(\bar{E}_{v}\right) \Subset X$ because it's dimension is one and it's genus is zero. From the condition, there is a holomorphic function $g$ on $f\left(\bar{E}_{v}\right)$ such that $g(r)=0$ and $g\left(f\left(z_{v}^{\prime}\right)\right)=1$ for fixed $v \geqq \max \left(N_{\varepsilon}, M_{\varepsilon}\right)$ where $\varepsilon$ is sufficiently small. Then $\left|g \circ f\left(\partial E_{v}\right)\right| \leqq \frac{1}{2}$ and $\left|g \circ f\left(E_{v}\right)\right| \leqq \frac{1}{2}$ by the maximum principle. This contradicts to $\left|g \circ f\left(z_{v}^{\prime}\right)\right|=1$.

Then $f\left(z_{v}\right) \rightarrow r$ for every $z_{v} \rightarrow z_{0}$ where $z_{v} \in \Delta-e$ and $f$ can be extended holomorphically at $z_{0}$ by Proposition 3.4. And $f$ can be extended holomorphically a map of $\Delta$ to $X$ because we take $z_{0} \in e$ arbitrarily.

Remark 4.2 Let $e$ be a logarithmic closed capacity zero set in $\Delta$. There is a simply connected domain $D$ surrounded by a closed Jordan curve where $D$ is near $\Delta$ such as $\Delta \supset D$ and $D \cap e$ is compact in $D$ from Corollary 2.5. Then there is a conformal map $\varphi$ of $D$ to $\Delta$ and $\varphi(e)$ is a compact logarithmic capacity zero set of $\Delta$. Therefore we may assume that above proposition holds good to such $D$.

Remark 4.3 In Proposition 4.1 if $f$ is a holomorphic injective map of $\Delta-e$ to $X, f\left(\bar{E}_{v}\right)$ is a bordered Riemann surface with genus 0 . Then it is easy to see that $f$ can be extended holomorphically to a map of $\Delta$ to $X$ which is an 
arbitrary open or compact hyperbolic space.

Proposition 4.4 Let $\Delta$ and $e$ be the same as in Proposition 4.1. Let $X$ be a compact hyperbolic space with an arbitrary dimension whose universal covering space $\tilde{X}$ is a Stein space. Then if $f$ is a holomorphic map of $\Delta-e$ to $X$, then $f$ can be extended holomorphically to a map of $\Delta$ to $X$.

Proof. We use the same notations in the proof of Proposition 4.1. We take a disjoint Jordan smooth curves $l_{1}, \cdots, l_{p}$ from points $z_{1}, \cdots, z_{p}$ on $C_{v}^{0}$ to $z_{1}^{1}, \cdots, z_{p}^{1}$ on $C_{v+1,1}^{1} \cdots C_{v+1, p}^{1}$ respectively in $\bar{E}_{v}$ where $C_{v+1}^{1}=C_{v+1,1}^{1} \cup \cdots \cup C_{v+1 . p}^{1}$ such that $E_{v}^{\prime}=E_{v}-\bigcup_{i=1}^{p} l_{i}$ is simply connected domain. Let $\pi: \tilde{X} \rightarrow X$ be a covering map and $g$ be a holomorphic function on $\tilde{X}$. The function $g \circ \pi^{-1} \circ f$ is a single valued function on $E_{v}^{\prime}$, it is a single valued function on $\overline{E_{v}}$ by Painlevé theorem and the nature of $f$. Since $\tilde{X}$ is Stein, we can take $g$ such that $g\left(\pi^{-1}(r)\right)=0$ and $g\left(\pi^{-1}\left(f\left(z_{v}^{\prime}\right)\right)=1\right.$. Then $f$ can be extended holomorphically to a map of $\Delta$ to $X$ from the proof of Proposition 4.1.

Theorem 4.5 Let $M$ be a manifold with an arbitrary dimension and $E$ be a closed capacity zero set of $M$. Let $X$ be the same as in Proposition 4.1. Then if $f$ is a holomorphic map of $M-E$ to $X$ such that $f(M-E)$ is relatively compact in $X$, in particular if $X$ is compact, $f$ can be extended holomorphically to a map of $M$ to $X$.

Proof. Let $\left(z_{0}\right)$ be an arbitrary point of $E$ and $\overline{D_{0}}$ be some closed neighborhood of $M$. Let $\overline{D_{1}}$ be some closed neighborhood of $\left(z_{0}^{\prime}\right)$ of $M$ such that $\left(z_{0}^{\prime}\right) \notin E$ and $\bar{D}_{1} \subset \overline{D_{0}}$ and $\left(z_{0}\right) \in D_{1}$ from Corollary 3.5. And we may assume that $D_{1}$ is biholomorphic to a closed unit ball $\bar{B}$ centered at $(0)$ which corresponds to $\left(z_{0}^{\prime}\right)$. We identify $\overline{D_{1}}$ to $\bar{B}$. Since every line $L$ through $(0)$ in $\bar{B}, \bar{B} \cap L \cap E$ is a closed logarithmic capacity zero set and $B \cap L=\Delta$ can be deformed a little to $D$ such that Proposition 4.1 holds good for $D$ from Remark 4.2. Then every line $L$ through $(0)$ in $\bar{B}$, we may consider that $f_{\left.\right|_{L}}$ is a holomorphic map of $\Delta$ to $X$. It is easy to see that $f$ is holomorphic on $B$ by blowing up method at $(0)$ and Hartogs theorem. Since we take $\left(z_{0}\right)$ as an arbitrary point of $E, f$ can be extended holomorphically to a map of $M$ to $X$.

Theorem 4.6 Let $M$ and $E$ be the same as in Theorem 4.5 and $X$ be the same of Proposition 4.4. Then if $f$ is an holomorphic map of $M-E$ to $X$, then $f$ can be extended holomorphically to a map of $M$ to $X$.

Proof. From Proposition 4.4 and the same method of Theorem 4.5, $f$ can be extended holomorphically to a map of $M$ to $X$.

Corollary 4.7 Let $M$ and $E$ be the same as in Theorem 4.5. If $f$ is a holomorphic map of $M-E$ to a compact Riemann surface $R$ whose genus $\geq 2, f$ can be extended holomorphically to a map of $M$ to $R$.

Proof. Since the universal covering of $R$ is a unit disk, above statement is true by Theorem 4.6.

From Remark 4.3 and the same method of Theorem 4.5 following corollary is obtained.

Corollary 4.8 Let $M$ be and $E$ be the same as in Theorem 4.5. If $f$ is a holomorphic injective map of $M-E$ to a compact hyperbolic space $X$ with an arbitrary dimension, $f$ can be extended holomorphically to a map of $M$ to $X$.

From above corollary it is easy to see the following theorem.

Theorem 4.9 Let $X$ be an arbitraly compact hyperboric manifold, $E$ be a closed capacity zero set of $X$ and $f \in \operatorname{Aut}(X-E)$. Then $f \in \operatorname{Aut}(X)$.

Problem 4.10 Let $X$ be a compact hyperbolic space. When is the universal covering space of $X$ a Stein space ?

\section{References}

Fujimoto, H. (1971). Riemann domains with boundary of capacity zero. Nagoya Math. J., 44, 1-15.

Kobayashi, S. (1998). Hyperbolic complex spaces. Grundlehlen der mathmatishen Wissenschaften, 318. Berlin, Heiderberg: Springer-Verlag. http://dx.doi.org/10.1007/978-3-662-03582-5

Kobayashi, S. (2005). Hyperbolic Manifolds and Holomorphic Mappings (2nd ed.). New York: Marcel Dekker. http://dx.doi.org/10.1142/5936

Nishino, T. (1979). Prolongments analytiques au sens de Riemann. Bull. Soc. Math. France, 107, 97-112.

Nishino, T. (2001). Function Theory in Several Complex Variables. Translations of Mathmatical Monographs, 193. Amer. Math. Soc., Providence, RI.

Noguchi, J. (1993). Some problemes in value distribution and hyperbolic manifolds. Kyoto Univ. R.I.M.S. Kokyuroku, 819, 66-73. 
Selberg, H. (1937). Über Puntmengen von Kapazität Null. Avh. Norske Vid. Acad. Oslo, 10, 1-10.

Suzuki, M. (1987). Comportement des applications holomorphes autour d'une ensemble polaire. C. R. Acad. Sc. Paris, 304, 191-194.

Suzuki, M. (1988). Comportement des applications holomorphes autour d'un ensaemble polaire, II. C. R. Acad. Sc. Paris, 306, 535-538.

\section{Copyrights}

Copyright for this article is retained by the author(s), with first publication rights granted to the journal.

This is an open-access article distributed under the terms and conditions of the Creative Commons Attribution license (http://creativecommons.org/licenses/by/3.0/). 